\title{
Anti-Inflammatory and Anti-Arthritic Efficacy and Safety of Purified Shilajit in Moderately Arthritic Dogs
}

\author{
Lawley $\mathrm{S}^{1}$, Gupta $\mathrm{RC}^{* 1}$, Goad JT ${ }^{1}$, Canerdy $\mathrm{TD}^{1}$ and Kalidindi $\mathrm{SR}^{2}$ \\ ${ }^{1}$ Murray State University, Breathitt Veterinary Center, Hopkinsville, KY, USA \\ ${ }^{2}$ Natreon Inc. New Brunswick, NJ, USA
}

*Corresponding author: Gupta RC, Professor and Head of Toxicology Department, Murray State University, Breathitt Veterinary Center, P.O. Box 2000; 715 North Drive, Hopkinsville, KY, 42240-2000, USA, Fax: (270) 886-4295, Tel: (270) 886-3959, E-mail: rgupta@murraystate.edu

Citation: Lawley S, Gupta RC, Goad JT, Canerdy TD, Kalidindi SR (2013) Anti-Inflammatory and AntiArthritic Efficacy and Safety of Purified Shilajit in Moderately Arthritic Dogs. J Vet Sci Anim Husb 1(3): 302. doi: 10.15744/2348-9790.1.302

Received Date: October 31, 2013 Accepted Date: December 12, 2013 Published Date: December 16, 2013

\begin{abstract}
The objective of this investigation was to evaluate the efficacy and safety of purified Shilajit in moderately arthritic dogs. Ten clientowned dogs in a randomized double-blinded study received either a placebo or Shilajit (500 mg) twice daily for a period of five months. Dogs were evaluated each month for physical condition (body weight, body temperature, heart rate, and respiration rate) and pain associated with arthritis (overall pain, pain from limb manipulation, and pain after physical exertion). Serum samples collected from these dogs were examined each month for biomarkers of liver (bilirubin, ALT, and AST), kidney (BUN and creatinine) heart and muscle (creatine kinase) functions. The findings of this study revealed that dogs receiving Shilajit (Group-II) showed a significant $(\mathrm{P}<0.05)$ reduction in pain from limb manipulation by day 60, and overall pain and pain after physical exertion by day 120 . Maximum pain reduction, using all three criteria, was observed on day 150. Pain level remained significantly unchanged in dogs receiving the placebo. Dogs in either group showed no significant change $(\mathrm{P}>0.05)$ in physical parameters or serum markers, suggesting that Shilajit was well tolerated by moderately arthritic dogs. It was concluded that Shilajit significantly $(\mathrm{P}<0.05)$ reduced pain in osteoarthritic dogs and markedly improved their daily life without any side effects.
\end{abstract}

Keywords: Purified Shilajit; Osteoarthritis in canine; Shilajit safety; Anti-arthritic nutraceutical

\section{Introduction}

Currently, there are more than 78 million dogs in the US, and every fifth adult dog suffers from arthritis [1]. Dogs suffer more frequently from osteoarthritis (a chronic and progressive disease) than any other form of arthritis. Osteoarthritis, also known as degenerative joint disease, is a functional disorder of the joint, characterized by a change in joint shape secondary to a loss of articular cartilage, osteophyte formation, subchondral sclerosis, bone marrow lesions and synovial proliferation, with subsequent alteration of mechanical properties that result in decreased stability, movement and loading [2-9]. In the early stages of osteoarthritis, there is a progressive depletion of the cartilage proteoglycan leading to a net loss of matrix from the cartilage [10]. This leads to a cascade of negative events, including changes in enzymatic cleavage of proteoglycans and an increase in minor collagen types leading to structural damage and deterioration of the cartilage. The breakdown of cartilage can be increased with certain enzymes including the matrix metalloproteinase enzymes (MMPs). The severity of cartilage lesions in osteoarthritis can be cor- related with the levels of collegenase (MMP-1) present [7-9]. The cascading events lead to increased friction and inflammation in the joints. Dogs suffering with osteoarthritis show stiffness of joints, crepitus, pain upon manipulation of the joint, periarticular swelling, palpable effusion, restriction in range of motion, lameness and pain and loss of function [7, 11-16]. The combination of history, physical exam, and radiograph can help diagnose osteoarthritis in a canine patient. Radiographs indicate osteophytosis and subchondral bone sclerosis that develops over time in osteoarthritic patients. MRI findings can reveal changes consistent with osteoarthritis in the cartilage $[17,18]$.

There are multiple factors which influence dogs to develop arthritis, such as genetic predisposition, trauma or injury to the bones or joints, aging, poor nutrition, obesity and environmental factors [1,19]. Often, large breed dogs (e.g. German Shepherds, Labrador Retrievers, Siberian Huskies, Rottweilers and others) are more prone to develop osteoarthritis than are small breeds [20-22]. 
Arthritic dogs show a variety of signs and symptoms, from limping to an inability to walk due to joint stiffness. Pain is the number one complaint [11-14]. The objectives in treating arthritis are to minimize joint pain by reducing the inflammation and slowing the progression of the cartilage damage and to increase joint flexibility. These goals are achieved by employing a variety of pharmaceuticals, nutraceuticals, disease modifying agents and physical therapy along with acupuncture [14, 23-26]. Cyclooxygenase (COX) inhibiting non-steroidal antiinflammatory drugs (NSAIDS, such as carprofen, deracoxib, etodolac, firocoxib, ketoprofen, meloxicam, robenacoxib, rofecoxib, tepoxalin, etc) are used with caution as they may cause some adverse effects, such as reduced appetite, vomiting, gastrointestinal bleeding and hepatic and renal damage and dysfunction [23, 27-32]. In a recent prospective (randomized, placebo-controlled, blinded) study, Monteiro-Steagall et al [33] found that the incidence of adverse effects was not statistically different between treated and control dogs.

Recently, dog owners and veterinarians have considered managing osteoarthritis in dogs with herbs and nutraceuticals. Today, in humans and animals, glucosamine and chondroitin sulfate are the most common nutraceuticals to provide building blocks to repair the cartilage and to minimize and slow down the progression of osteoarthritis to ease inflammatory pain $[24,25,34-37]$.

Shilajit is a blackish brown exudate from the sedimentary rocks of the pristine himalayan mountains at about $10,000 \mathrm{ft}$ altitude. Purified shilajit is prepared from this exudate by a proprietary extraction process. the main constituents of shilajit are dibenzo- $\alpha$-pyrones(DBPs), DBP-chromoproteins and fulvic acids with DBP core. On the other hand, alluvial(plant based) fulvic acids do not have DBP core and do not have the same therapeutic efficacy as shjilajit. In fact, alluvial fulvic acids are used as fertilizers. Shilajit exerts many important roles in biological systems: (1) improves bioavailability of minerals and nutrients, (2) provides electrolytes, (3) detoxifies toxic substances, including heavy metals, (4) improves immune system and (5) exhibits antioxidant properties. Shilajit has been used for more than 3000 years for both preventative health and for treating many diseases in humans, including diabetes, arthritis, hypertension, immune dysfunction and loss of memory [3840]. The present study was undertaken to discover the therapeutic efficacy and safety of purified Shilajit in moderately arthritic dogs. The hypothesis of the present investigation was that purified Shilajit will provide ani-inflammatory and antiarthritic effects in moderately arthritic dogs with minimal side effects. In this paper, we present the findings of Shilajit as an effective and safe supplement that exerts anti-inflammatory and anti-arthritic effects in osteoarhtritic dogs. Shilajit appears very promising and offers an alternative to many other previously reported pharmaceuticals and nutraceuticals.

\section{Materials and methods}

40-65 pounds, were used in this study. These dogs, based on signs of joint stiffness, lameness and radiographic evidence, had pain at the level of moderate arthritis. Our inclusion criteria of dogs for this study excluded those having liver, kidney or heart disease, neoplasia, cancer or any other major disease. Institutional Animal Care and Use Committee (IACUC) approval and owner consents were obtained prior to the initiation of this study.

\section{Experimental design}

In a randomized double-blinded study, ten client-owned dogs received a placebo (Group-I) or Shilajit (Group-II). The placebo group contained only two dogs because IACUC advised us to keep to the number of dogs to a minimum due to the fact that arthritic dogs suffer from pain for a period of five months. Moreover, findings from our previous studies revealed that dogs receiving placebo showed no improvement in arthritis pain [11-14]. Eight dogs in Group-II received purified Shilajit $(500 \mathrm{mg}$ ) twice daily (one capsule before morning meal and one capsule before evening meal) for a period of five months. None of the dogs received any treatment or supplement for 3 to 4 weeks prior to the study or during the study period.

\section{Pain measurement}

At pre-determined intervals (i.e. 30 days), each dog was evaluated for overall pain, pain upon limb manipulation and pain after physical exertion, for a period of five months. Overall pain, on a scale of 1-10, was graded as: 0 , no pain: 2.5 , mild pain: 5 , moderate pain: 7.5 , severe pain: 10 , severe and constant pain. Pain after limb manipulation, on a scale of 0-4, was evaluated as: no pain: 0 , mild pain: 1 , moderate pain: 2 , severe pain: 3 , severe and constant pain: 4 . Pain after physical exertion, on a scale of $0-4$, was evaluated as: no pain: 0 , mild pain: 1 , moderate pain: 2 , severe pain: 3 , and severe and constant pain: 4 . The physical examination of each limb started with the forelimbs and ended with the rear limbs. The evaluation focused on manipulation of the limbs in a forward, backward and circular motion. Three main joints in each limb included for evaluation were the shoulder joint, knee joint and stifle joint. Popping and cracking of the joint as well as vocal pain were noted for each canine. Detailed criteria of the measurement of pain are provided in our earlier publications [11-14]. The present investigation was carried out on moderately arthritic dogs. A moderately arthritic dog exhibits overall pain of about 5 on a scale of 1-10; pain upon limb manipulation about 2 on a scale of 1-4; and pain after physical exertion about 2 on a scale of 1-4.

\section{Physical examination}

On a monthly basis, dogs were given a full physical examination for body weight, body temperature and heart rate. Normal values for these parameters are mentioned below Table 1 .

\section{Animals}

Ten client-owned moderately arthritic dogs, weighing between 


\begin{tabular}{|l|l|l|l|l|l|l|l|l|}
\hline Parameter & Unit & Placebo/Treated & Day 0 & Day 30 & Day 60 & Day 90 & Day 120 & Day 150 \\
\hline \multirow{2}{*}{ Body Weight } & \multirow{2}{*}{ 1b } & Placebo & $54.1 \pm 11.05$ & $54.0 \pm 10.8$ & $53.4 \pm 9.85$ & $54.6 \pm 8.95$ & $53.6 \pm 9.95$ & $53.7 \pm 9.5$ \\
\cline { 3 - 8 } & & Treated & $54.5 \pm 3.37$ & $54.9 \pm 3.53$ & $54.4 \pm 3.26$ & $54.9 \pm 3.16$ & $54.5 \pm 3.22$ & $54.9 \pm 3.27$ \\
\hline \multirow{2}{*}{ Heart Rate } & \multirow{2}{*}{ Beats/min } & Placebo & $135 \pm 15$ & $120 \pm 0$ & $115 \pm 5$ & $90 \pm 10$ & $98 \pm 2$ \\
\cline { 3 - 8 } & & Treated & $106.8 \pm 4.96$ & $117.6 \pm 5.89$ & $96.5 \pm 6.47$ & $102.4 \pm 3.40$ & $102.8 \pm 4.60$ & $108.9 \pm 2.81$ \\
\hline \multirow{2}{*}{ Body Temperature } & \multirow{2}{*}{$\mathrm{F}$} & Placebo & $100.3 \pm 5.41$ & $100.3 \pm 0.2$ & $99.9 \pm 0.65$ & $100.3 \pm 0.2$ & $100.4 \pm 0.3$ & $100.1 \pm 1.2$ \\
\cline { 3 - 8 } & & Treated & $100.1 \pm 0.43$ & $99.6 \pm 0.26$ & $99.6 \pm 0.23$ & $99.6 \pm 0.29$ & $100.9 \pm 0.30$ & $100.4 \pm 0.20$ \\
\hline
\end{tabular}

Values are means \pm SEM; Normal heart rate $=70-160$ beats per minute; Normal body temperature $=101-102.5 \circ$.

Table 1: Effects of placebo or Shilajit (500 mg, bid) on physical parameters in moderately arthritic dogs

\begin{tabular}{|c|c|c|c|c|c|c|c|c|}
\hline Parameter & Unit & Placebo/Treated & Day 0 & Day 30 & Day 60 & Day 90 & Day 120 & Day 150 \\
\hline \multirow[t]{2}{*}{ BUN } & \multirow[t]{2}{*}{$\mathrm{mg} / \mathrm{dl}$} & Placebo & $19.5 \pm 3.5$ & $18 \pm 1$ & $22 \pm 2$ & $21 \pm 1$ & $17 \pm 1$ & $17 \pm 4$ \\
\hline & & Treated & $17.1 \pm 2.88$ & $15.6 \pm 1.78$ & $18.1 \pm 2.30$ & $18.1 \pm 2.30$ & $20.5 \pm 2.85$ & $20.8 \pm 2.64$ \\
\hline \multirow[t]{2}{*}{ Creatinine } & \multirow[t]{2}{*}{$\mathrm{mg} / \mathrm{dl}$} & Placebo & $0.92 \pm 0.16$ & $0.75 \pm 0.02$ & $0.72 \pm 0.02$ & $0.76 \pm 0.09$ & $0.81 \pm 0.02$ & $0.82 \pm 0.03$ \\
\hline & & Treated & $1.00 \pm 0.09$ & $0.79 \pm 0.03$ & $0.79 \pm 0.04$ & $0.79 \pm 0.09$ & $0.92 \pm 0.05$ & $0.94 \pm 0.04$ \\
\hline \multirow[t]{2}{*}{ Total bilirubin } & \multirow[t]{2}{*}{$\mathrm{mg} / \mathrm{dl}$} & Placebo & $0.5 \pm 0.3$ & $0.2 \pm 0$ & $0.3 \pm 0.1$ & $0.35 \pm 0.05$ & $0.2 \pm 0$ & $0.3 \pm 0.1$ \\
\hline & & Treated & $0.21 \pm 0.04$ & $0.25 \pm 0.04$ & $0.23 \pm 0.05$ & $0.39 \pm 0.09$ & $0.26 \pm 0.04$ & $0.31 \pm 0.04$ \\
\hline \multirow[t]{2}{*}{ ALT } & \multirow[t]{2}{*}{$\mathrm{IU} / \mathrm{L}$} & Placebo & $45.5 \pm 23.5$ & $35.5 \pm 8.5$ & $28 \pm 0$ & $40 \pm 12$ & $27 \pm 0$ & $57.5 \pm 14.5$ \\
\hline & & Treated & $47.9 \pm 4.54$ & $59.9 \pm 8.41$ & $75.1 \pm 20.4$ & $57.6 \pm 4.59$ & $60.8 \pm 7.36$ & $58.6 \pm 4.95$ \\
\hline \multirow[t]{2}{*}{ AST } & \multirow[t]{2}{*}{$\mathrm{IU} / \mathrm{L}$} & Placebo & $127.5 \pm 39.5$ & $123 \pm 33$ & $84 \pm 4$ & $104 \pm 15$ & $779.5 \pm 629.5$ & $166.4 \pm 25.7$ \\
\hline & & Treated & $198.4 \pm 44.7$ & $349 \pm 80.9$ & $224.6 \pm 66.2$ & $132.0 \pm 28.2$ & $246.0 \pm 84.8$ & $166.4 \pm 25.7$ \\
\hline \multirow[t]{2}{*}{ CK } & \multirow[t]{2}{*}{$\mathrm{IU} / \mathrm{L}$} & Placebo & $28.5 \pm 3.5$ & $22 \pm 7$ & $19.5 \pm 5.5$ & $23.5 \pm 5.5$ & $45.5 \pm 26.5$ & $92.5 \pm 3.5$ \\
\hline & & Treated & $27.1 \pm 3.26$ & $31.5 \pm 3.13$ & $28.4 \pm 3.57$ & $28.5 \pm 2.35$ & $30.5 \pm 3.59$ & $29.1 \pm 3.60$ \\
\hline
\end{tabular}

Values are means \pm SEM; Normal reference values: BUN (7-26 mg/dl); Creatinine (0.0-1.35 mg/dl); Total bilirubin (0.1-0.6 mg/dl); ALT (10-120 IU/L); AST (15-65 IU/L); CK (60-450 IU/L).

Table 2: Effects of placebo or Shilajit (500 mg, bid) on serum biomarkers of liver (bilirubin, ALT, and AST), kidney (BUN and creatinine), heart and skeletal muscle (CK) functions in moderately arthritic dogs

\section{Serum biomarkers assays}

Blood samples were collected from the cephalic vein using a $3 \mathrm{~mL}$ syringe with a 22 gauge needle and were stored in a marble top tube. Samples were then spun to collect serum and transferred to a red top tube for evaluation. Serum samples were collected each month and analyzed for liver (bilirubin, ALT, AST), kidney (BUN and creatinine), heart and muscle (CK) functions, using a Beckman AU 480 serum analyzer. The serum sample assay indicated that neither placebo nor Shilajit produced adverse effects in vital organs of arthritic dogs. Normal reference values of all serum parameters are provided below Table 2.

\section{Statistical analysis}

The data presented are means \pm SEM. Statistical significance of difference was determined by analysis of variance (ANOVA) coupled with Tukey-Kramer test $(\mathrm{P}<0.05)$ using the NCSS2000 Statistical Analysis and Graphics Software for Windows ${ }^{\oplus}$.

\section{Results}

In this double-blinded clinical trial, moderately arthritic dogs receiving placebo (Group-I) or purified Shilajit $(500 \mathrm{mg}$ ) twice daily for a period of five months, were evaluated for physical parameters, pain level and serum biomarkers of liver, kidney, heart and muscle functions.

On a monthly basis, each dog was examined for pain level (overall pain, pain after limb manipulation and pain after physical exertion) and the data are shown in Figures 1-3. While evaluating overall pain, the key points were to observe the dog's gait, range of motion, ability to sit or lie down, ability to rise from a sitting position, ability to rise from a lying position, and posture while standing. Group-II dogs receiving Shilajit showed significant $(\mathrm{P}<0.05)$ reduction in overall pain by day $120(3.00 \pm 0.27)$ compared to day $0(5.38 \pm 0.75)$. The maximum reduction in overall pain was noted on day $150(2.38 \pm 0.32)$. Dogs receiving placebo showed no significant change in overall pain.

Pain after limb manipulation was measured in each limb of the dog (placed in lateral recumbency) for flexibility, joint integrity, crepitance and vocalization. The pain level was significantly reduced by day $60(1.63 \pm 0.18)$ and the trend continued until day 150 when the pain level was maximally reduced (1.25 \pm 0.31$)$ (Figure 2). Pain after limb manipulation remained significantly unchanged in Group-1 (placebo) dogs. Figure 3 shows the data of pain after physical exertion in dogs receiving placebo or Shilajit. The canines were evaluated for pain after three minutes of jogging. After jogging, pain level was assessed 
based on the dog's unwillingness to move, body position, limping, flexibilty, joint integrity, crepitance and vocalization. Significant reduction in pain after physical exertion was noted on day $120(1.37 \pm 0.26)$ compared to day $0(2.63 \pm 0.26)$ in dogs receving Shilajit. The maximum reduction in pain was observed on day 150 (1.25 \pm 0.28$)$. Dogs on placebo showed no significant change in pain level.

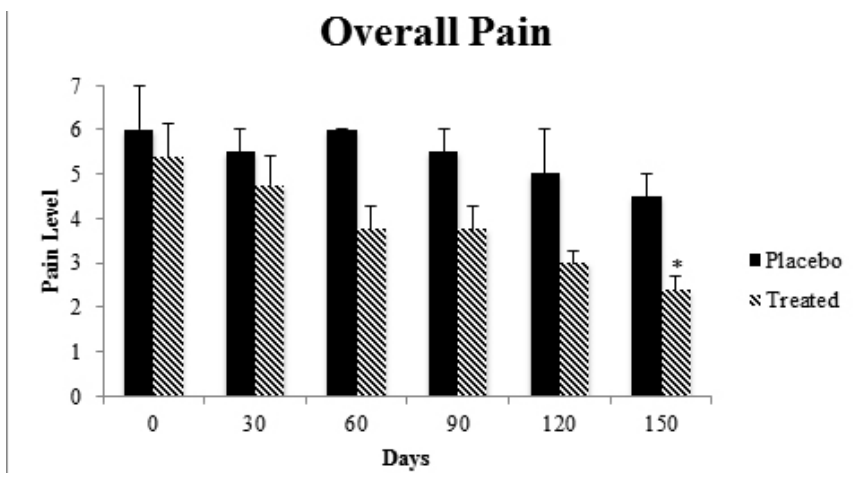

${ }^{*}$ Significantly different compared to pre-treated values $(\mathrm{P}<0.05)$.

Figure 1: Effects of placebo or Shilajit $(500 \mathrm{mg}$, bid) on overall pain in moderately arthritic dogs. Overall pain was graded on a scale of $0-10(0$, no pain; 2.5 , mild pain; 5 , moderate pain; 7.5 , severe pain; and 10 , severe and constant pain). Details of overall pain measurement criteria are described in the text and our previous publications [11-14].

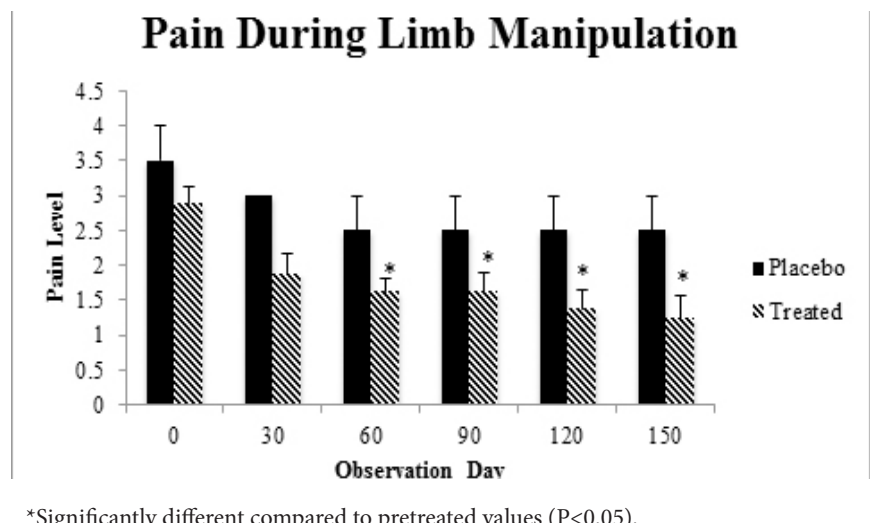

Figure 2: Effects of placebo or Shilajit (500 mg, bid) on pain from limb manipulation in moderately arthritic dogs. Pain from limb manipulation was graded on a scale of $0-4$ ( 0 , no pain; 1 , mild pain; 2 , moderate pain; 3 , severe pain; and 4 , severe and constant pain). Details of pain measurement after limb manipulation criteria are described in the text and our previous publications [11-14].

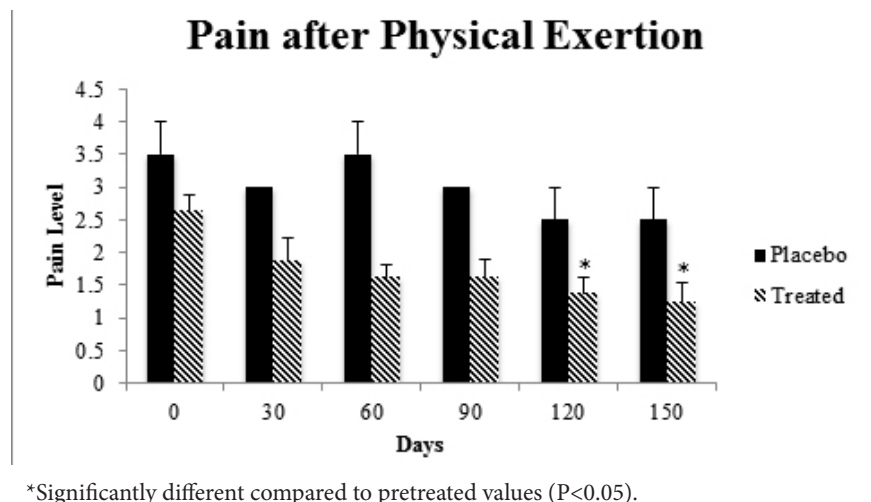

Figure 3: Effects of placebo or Shilajit $(500 \mathrm{mg}$, bid) on pain after physical exertion in moderately arthritic dogs. Pain after physical exertion was graded on a scale of $0-4$ ( 0 , no pain; 1 , mild pain; 2 , moderate pain; 3 , severe pain; and 4 , severe and constant pain). Details of pain measurement after physical exertion criteria are described in the text and our previous publications [11-14].
Data of physical parameters (body weight, body temperature, and heart rate are shown in Table 1. Dogs receiving placebo or Shilajit showed no significant change in any physical parameters during the course of this investigation. Table 2 presents serum chemistry data for biomarkers of liver (bilirubin, ALT, and AST), kidney (BUN and creatinine), heart and muscle (CK) functions. Dogs receiving placebo or Shilajit showed no significant change in serum biomarkers during the study period of 150 days.

\section{Discussion}

Osteoarthritis is prevalent in dogs primarily due to aging, but it can also be due to obesity, poor nutrition, injury, genetic predisposition and other environmental factors [1, 19-22]. Dogs are also affected by osteoarthritis, a progressive degenerative disease characterized by loss of cartilage. Eventually, dogs suffer from pain and poor quality of life due to immobility.

Veterinarians have a wide range of options to treat and manage osteoarthritis, including surgery, NSAIDS, physical therapy with acupuncture, nutraceuticals and other disease modifying agents $[13,14,23-26,41]$.

Currently, glucosamine and chondroitin are the two most commonly used nutraceuticals to manage osteoarthritis in dogs, but their efficacy is limited $[24,35]$. In the last ten years, we have evaluated the therapeutic efficacy and safety of many nutraceuticals in arthritic dogs [11-14]. In a most recent chronic study, we found that Crominex $3+$ (a mixture of trivalent chromium, Phyllanthus emblica extract and purified Shilajit) was very effective in ameliorating arthritic pain in dogs without causing any untoward events [42].

In the present paper, we report that Shilajit alone at a higher dose $(500 \mathrm{mg}$, bid) compared to that present in Crominex $3+$ $(7.5 \mathrm{mg}$, bid), is equally effective in reducing arthritic pain and enhancing the daily activity of dogs without exerting any side effects. Shilajit administration ameliorated arthritic pain in all three categories (overall pain, pain after limb manipulation, and pain after physical exertion) with maximum effect noted on day 150. Shilajit is a pale-brown to blackish-brown resinous substance and is a herbo-mineral complex compound that consists of humus and organic plant (Styrax officinalis, Trifolium repens and others) materials. By having many bioactive minerals and plant active principles, such as fulvic acid (low molecular weight substances like uronic acids, phenolic glycosides and amino acids), humic acid (low molecular weight substances like phenolic acids, anti-oxidants and free radical scavengers) and free and conjugated dibenzo-alpha-pyrones, Shilajit is known to exert multiple pharmacological actions. Some of these actions include antibacterial, anti-arthritic, anti-ulcerogenic, anti-hyperglycemic, immunomodulatory, anti-inflammatory, anti-radical, spermatogenic and ovogenic, anti-aging and energetic properties [28, 38, 40, 43-47]. Shilajit has been used for both preventative health and for treating many diseases (such as allergies, diabetes, hypertension, loss of memory, immune dysfunction, arthritis, loss of libido, etc) for more than 3000 years. 
Since Shilajit has multiple bioactive principles, it is highly likely that it reduced the arthritic pain due to a variety of biochemical and pharmacological mechanisms, including antiinflammatory, anti-oxidant, immune-modulatory and energetic properties.

\section{Conclusions}

In conclusion, Shilajit is an all-natural supplement which offers significant anti-arthritic properties including reduction of pain and inflammation. All dogs responded well to Shilajit administration without exhibiting any untoward effects, thereby giving this supplement an edge over many other nutraceuticals and pharmaceuticals.

\section{Acknowledgements}

The authors would like to thank Ms. Robin B. Doss and Ms. Michelle A. Lasher for their technical assistance in preparation of this manuscript.

\section{References}

1) Arthritis Foundation (2013). Spotting Arthritis in Spot. Retrieved 09 2013, 02, from Arthritis Foundation.

2) Vaughan-Scott T, Taylor JH (1997). The pathophysiology and medical management of canine osteoarthritis. J S Afr Vet Assoc. 68: 21-25.

3) Bellamy N, Carr A, Dougados M, Shea B, Wells G (2001). Towards a definition of "differences" in osteoarthritis. J Rheumatol. 28: 427-430.

4) Lennon E, Marcellin-Little D (2005). Canine Osteoarthritis. Retrieved August 5, 2013 from Arthritis M.D.

5) Burns K (2006). Research targets and conditions of older cats and dogs. J Am Vet Med Assoc. 229: 482-483.

6) Centers for Disease Control and Prevention (CDC) Arthritis-Basic-Definition-Osteoarthritis. Retrieved June 18, 2010.

7) Renberg WC (2005). Pathophysiology and management of arthritis. Vet Clin Small Anim. 35: 1073-1091.

8) Dore D, Martens A, Quinn S, Ding C, Winzenberg C, et al.(2010) Bone marrow lesions predict site-specific cartilage defect development and volume loss: a prospecitve study in older adults. Arthr Res Ther. 12: R222.

9) Umlauf D, Frank S, Pap T, Bertrand J (2010) Cartilage, biology, pathology, and repair. Cell Mol Life Sci. 67: 4197-4211.

10) Reid DM, Miller GC (2008). Clinical Trails in Rheumatoid Arthritis and Osteoarthritis.Aberdeen, UK: Springer-Verlag London Limited.

11) Deparle LA, Gupta RC, Canerdy TD, Goad JT, D’Altilio M, et al. (2005). Efficacy and safety of glycosylated undenatured type-II collagen (UC-II) in therapy of arthritic dogs. J Vet Pharmacol Ther. 28: 385-390.

12) D’Altilio M, Peal A, Alvey M, Simms C, Curtsinger A, et al. (2007). Therapeutic efficacy and safety of undenatured type II collagen singly or in combination with glucosamine and chondroitin in arthritic dogs. Toxicol Mechan Methods.17:189-196.

13) Peal A, D’Altilio M, Simms C, Alvey M, Gupta RC, et al. (2007). Therapeutic efficacy and safety of undenatured type-II collagen (UC-II) alone or in combination with (-) hydroxycitric acid and chromemate in arthritic dogs. J Vet Pharmacol Ther. 30: 275-278.

14) Gupta RC, Canerdy TD, Lindley J, Konemman M, Minniear J, et al. (2012) Comparative therapeutic efficacy and safety of type-II collagen (UC-II), glucosamine and chondroitin in arthritic dogs: pain evaluation by ground force plate. J Anim Physiol Anim Nutri. 96: 770-777.

15) Osteoarthritis in Dogs-Signs and Symptoms of Arthritis and Osteoarthritis. (2012) Retrieved August 8, 2013, from Osteoarthritis in Dogs.
16) Fahie MA, Ortolano GA, Guercio V, Schaffer JA, et al. (2013). A randomized controlled trial of the efficacy of autologous platelet therapy for the treatment of osteoarthritis. J Am Vet Med Assoc. 243:1291-1297.

17) Kumar D, Wyatt CR, Lee S, Nardo L, Link TM, et al. (2013). Association of cartilage defects, and other MRI findings with pain and function in individuals with mild-moderate radiographic hip osteoarthritis and controls. Osteoarthr Cartil. 21: 1685-1692.

18) Mosher TJ, Walker EA, Petscavage-Thomas J, Guermazi A (2013). Osteoarthritis year 2013 in review: Imaging. Osteoarthr Cartil. 21: 1425-1435.

19) Global Animal (2012). Stiff Joints? How To Treat Dog Arthritis . Retrieved February 18, 2013, from Global Animal.

20) Richardson DC, Schroenherr WD, Zicker SC (1997). Nutritional management of osteoarthritis. Vet Clin North Am, Small Anim Pract. 27: 883-911.

21) Elliot D (2007) Nutritional management of canine arthritis. Vet Focus 17: 43-48.

22) ASPCA (2013) Hip Dysplasia. Retrieved 9 13, 2013, from ASPCA.

23) Moreau M, Dupuis J, Bonneau NH, Desnoyers M (2003). Clinical evaluation of a nutraceutical, carprofen and meloxicam for the treatment of dogs with osteoarthritis. Vet Rec. 152: 323-329.

24) Neil KM, Caron JP, Orth MW (2005). The role of glucosamine and chondroitin sulfate in treatment and prevention of osteoarthritis in animals. J Am Vet Med Assoc. 226:1079-1088.

25) McCarthy G, O’Donovan J, Jones B (2007). Randomized double-blind, positive -controlled trial to assess the efficacy of glucosamine/chondroitin sulfate for the treatment of dogs with osteoarthritis. Vet J. 174: 54-61.

26) Sawaya S (2007). Physical and altenative therapies in the management of arthritic patients. IVIS Vet Focus 17: 37-42.

27) Bergh MS, Budsberg SC. (2005). The coxib NSAIDs : potential clinical and pharmacologic importance in veterinary medicine. J Vet Int Med. 19: 633-643. 28) Sessions JK, Reynolds LR, Budsberg SC (2005). In vivo effects of carprofen, deracoxib, and etodolac on prostanoid production in blood, gastric mucosa, and synovial fluid in dogs with chronic osteoarthritis. Am J Vet Res. 66: 812817.

29) Raekillio M, Hielm-Bjorkman A, Kejonen J, Salonen H, Sankari SM (2006). Evaluation of adverse effects of long-term orally administered carprofen in dogs. J Am Vet Med Assoc. 228: 876-880.

30) Canine Arthritis (2006) Retrieved 07 2013, from Canine Arthritis.

31) Innes JF, Clayton J, Lascelles B (2010). Review of the safety and efficacy of long- term NSAID use in the treatment of canine osteoarthritis. Vet Rec. 166 (8): 226-230.

32) Rychel J (2010). Diagnosis and Treatment of Osteoarthritis. Topics Compan Anim Med. 25: 20-25.

33) Monteiro-Steagall BP, Steagall PV, Lascelles BD (2013). Systematic review of nonsteroidal anti-inflammatory drug-induced adverse effects in dogs. J Vet Int Med. 27: 1011- 1019.

34) Lee YH, Woo JH, Choi SJ, Ji JD, Song GG (2010). Effect of glucosamine or chondroitin on the osteoarthritis progression: a meta-analysis. Rheumatol Int. 30: 357-360.

35) Canine Osteoarthritis Management with Glucosamine for Dogs (2012). Retrieved 8 19, 2013

36) Ohnishi A, Osaki T, Matahira Y, Tsuka T, Imagawa T (2013). Correlation of plasma amino acid concentrations and chondroprotective effects of glucosamine and fish collagen peptide on the development of osteoarthritis. J Vet Med Sci. 75: 497-502.

37) Iovu M, Dumais G, Du Souich P (2008). Anti-inflammatory activity of chondroitin sulfate. Osteoarthr Cartilage 16: S14-18.

38) Goel RK, Banerjee RS, Acharya SB (1990). Antiulcerogenic and antiinflammatory studies with shilajit. J Ethnopharmacol. 29: 95-103.

39) Bhattacharya SK, Ghosal S (1992). Effect of Shilajit on rat brain monoamines. Phytother Res. 6: 163-164.

40) Agarwal SP, Khanna R, Karmarker R, Anwar K, Khar RK (2007). Shilajit: a review. Phytother Res. 21(5):401-405.

41) Veterinary Medical and Surgical Group. (2013). Osteoarthritis.

42) Fleck A, Goad JT, Gupta RC, Lasher MA, Canerdy TD, Kalidindi SR (2013) Efficacy and safety of Crominex 3+(trivalent chromium, Phyllanthus emblica extract, and Shilajit) in moderately arthritic dogs. To be Submitted.

43) Acharya SB, Frotan MH, Goel RK, Tripathy SK, Das PK (1988). Pharmacological actions of Shilajit. Indian J Exp Biol. 26: 775-777. 
44) Bhattacharya SK, Sen AP, Ghosal S (1995). Effects of Shilajit on biogenic free radicals. Phytother Res 9: 56-59.

45) Tripathi YB, Shukla S, Chaurasia S, Chaturvedi S (1996). Antilipid peroxidative property of Shilajit. Phytother Res. 10: 269-270.
46) Park JS, Kim GY, Han K (2006). The spermatogenic and ovogenic effects of chronically administered Shilajit to rats. J Ethnopharmacol. 107: 349-353.

47) Meena H, Pandey HK, Arya MC, Ahmed Z (2010). Shilajit: A panacea for high-altitude problems. Int J Ayurveda Res, 1 (1): 37-40.

\footnotetext{
Submit your next manuscript to Annex Publishers and benefit from:

Dasy online submission process

> Rapid peer review process

> Online article availability soon after acceptance for Publication

Open access: articles available free online

> More accessibility of the articles to the readers/researchers within the field

Better discount on subsequent article submission

Submit your manuscript at

http://www.annexpublishers.com/paper-submission.php
} 